\title{
Direct Numerical Simulation of Disperse Multiphase High-Speed Flows
}

Nourgaliev, R. R., Dinh, T. N., Theofanous, T. G., Koning, J. M., Greenman, R. M., Nakafuji, G. T.

February 19, 2004

42nd AIAA Aerospace Sciences Meeting and Exhibit Reno, NV, United States January 5, 2004 through January 8, 2004 
This document was prepared as an account of work sponsored by an agency of the United States Government. Neither the United States Government nor the University of California nor any of their employees, makes any warranty, express or implied, or assumes any legal liability or responsibility for the accuracy, completeness, or usefulness of any information, apparatus, product, or process disclosed, or represents that its use would not infringe privately owned rights. Reference herein to any specific commercial product, process, or service by trade name, trademark, manufacturer, or otherwise, does not necessarily constitute or imply its endorsement, recommendation, or favoring by the United States Government or the University of California. The views and opinions of authors expressed herein do not necessarily state or reflect those of the United States Government or the University of California, and shall not be used for advertising or product endorsement purposes.

This work was done in part under the auspices of the U.S. Department of Energy by the University of California, Lawrence Livermore National Laboratory Under Contract No. W-7405-Eng-48. 


\title{
Direct Numerical Simulation of Disperse Multiphase High-Speed Flows
}

\author{
R.R.Nourgaliev, T.N.Dinh, and T.G.Theofanous \\ Center for Risk Studies and Safety, University of California, Santa Barbara \\ J.M.Koning, R.M.Greenman, and G.T.Nakafuji \\ Lawrence Livermore National Laboratory
}

\begin{abstract}
A recently introduced Level-Set-based Cartesian Grid (LSCG) Characteristics-Based Matching (CBM) method is applied for direct numerical simulation of shock-induced dispersal of solid material. The method incorporates the latest advancements in the level set technology and characteristics-based numerical methods for solution of hyperbolic conservation laws and boundary treatment. The LSCG/CBM provides unique capabilities to simulate complex fluid-solid (particulate) multiphase flows under high-speed flow conditions and taking into account particle-particle elastic and viscoelastic collisions. The particular emphasis of the present study is placed on importance of appropriate modelling of particle-particle collisions, which are demonstrated to crucially influence the global behavior of high-speed multiphase particulate flows. The results of computations reveal the richness and complexity of flow structures in compressible disperse systems, due to dynamic formation of shocks and contact discontinuities, which provide an additional long-range interaction mechanism in dispersed high-speed multiphase flows.
\end{abstract}

\section{Introduction}

$\mathbf{U}$ NDERSTANDING the physics of shock waves interacting with particle clouds is crucial in the development and analysis of several important technological and natural processes. These include explosive dispersal of solid material, ${ }^{22}$ shock-induced powder compaction and fluidization, ${ }^{20}$ protection of structures against explosions using particle layers or foams, supersonic combustion and explosions, safety of chemical plants, snow avalanches and others.

In the past, significant efforts have been invested in the development and application of direct numerical simulation (DNS) methods for low-speed incompressible fluid-solid (particulate) flows, which include Arbitrary Lagrangian-Eulerian (ALE) moving-mesh projection-based techniques, ${ }^{6,11}$ finite-element-based moving mesh techniques ${ }^{7}$ and lattice-Boltzmann(LBE)-based approaches. ${ }^{1,8}$ These DNS studies helped to extend fundamental understanding of rheology and dynamics in colloidal suspension.

In the case of high-speed compressible flows, past research had mainly been focused on experimental study of dusty gases and behavior of individual particles. Investigations of dense particle flows are few, related experimental data are scarce and largely qualitative. ${ }^{2,20}$ To our knowledge, there are no DNS studies available in an open literature.

The purpose of the present study is to investigate numerically the shock-particle interactions, aiming to extend the basic understanding of shock wave propagation in particle media, particles' collective behavior under shock waves, and shock-induced dispersal. The detailed knowledge about dynamics of particle cloud fluidization and pattern formation is indispensable for validation of hypotheses involved in construction of averaged ("effective field") equations and related constitutive physics.

For this, we bring to bear a newly developed numerical method named the "Level-Set-Based Cartesian Grid Characteristics-Based Matching (LSCG/CBM)" method. ${ }^{12,13}$ The LSCG/CBM method was developed for efficient simulation of complex moving boundaries and interfaces in compressible fluid flows. The method capitalizes on the latest advancements in the levelset technology ${ }^{17}$ (i.e., the PDE-based re-initialization and extension, ${ }^{18}$ the Ghost Fluid Method ${ }^{5}$ ), incorporates the latest generation of the high-order-accurate Godunov-based numerical schemes, ${ }^{21}$ and utilizes an innovative the third-order-accurate MUSCL-based implicit time discretization scheme, ${ }^{16}$ allowing to completely eliminate the "small-cell" issue, which plagues traditional Cartesian Grid methods. ${ }^{19}$ Application of the characteristics-based approach for boundary conditions allows for robust and accurate treatment of interfaces, respecting the information flow nearby. A novel particle-particle collision model developed previously ${ }^{15}$ opens possibility to simulate both elastic and viscoelastic collisions, allowing to delineate the effect of dissipative collision dynamics on global behavior of high-speed multiphase fluid-solid dispersed systems.

Here, the LSCG/CBM method is applied for DNS 
study of strong shock wave interactions with dense disperse fluid-solid multiphase systems. This includes interactions of shock with individual solid particles and clusters of solid particles. The particular focus is placed on investigation of the global dynamics of disperse multiphase fluid-solid system, effects of particleparticle collisions and particle's collective behavior.

The paper is organized as following. First, we will describe mathematical and physical model for DNS of compressible fluid-particle flows. Second, we will briefly introduce the numerical approach, followed by the summary of the method's validation. Next, the LSCG/CBM method will be applied to the simulation of the shock-induced collisions of two isolated particles. Finally, we will study interactions of the shock with isolated cluster of particles.

\section{Governing Equations}

In this section, we will describe mathematical and physical model for direct numerical simulation of the compressible fluid - solid particle multiphase flows.

\section{Fluid Dynamics}

The governing equations of compressible fluid dynamics can be written in the following generic form ${ }^{1}$ :

$$
\mathbf{U}_{t}+[\overrightarrow{\mathcal{F}}(\mathbf{U})]_{x}+[\overrightarrow{\mathcal{G}}(\mathbf{U})]_{y}+[\overrightarrow{\mathcal{H}}(\mathbf{U})]_{z}=\mathbf{S}(t, \mathbf{x}, \mathbf{U})
$$

where $t$ is time; $\mathbf{x}=(x, y, z)$ is a position-vector; $\mathbf{U}=\left(\mathrm{U}_{1}, \ldots, \mathrm{U}_{q}\right)^{\top}$ and $(\overrightarrow{\mathcal{F}}, \overrightarrow{\mathcal{G}}, \overrightarrow{\mathcal{H}})$ are the vector of the conservation variables and the vector of physical fluxes in $x, y$ and $z$ directions respectively; $q$ is a total number of the conservation variables. The Jacobian matrices $\mathbb{A}^{(\mathrm{x})}=\partial \overrightarrow{\mathcal{F}} / \partial \mathbf{U}, \mathbb{A}^{(\mathrm{y})}=\partial \overrightarrow{\mathcal{G}} / \partial \mathbf{U}$ and $\mathbb{A}^{(\mathrm{z})}=\partial \overrightarrow{\mathcal{H}} / \partial \mathbf{U}$ have $q$ real eigenvalues and a complete set of eigenvectors. The vector of source terms $\mathbf{S}(t, \mathbf{x}, \mathbf{U})$ is a function of time, space and conservation variables, in the most general case. We will consider Navier-Stokes $(N S)$ equations. In this case, the vectors of the conservation variables and fluxes are:

$$
\begin{aligned}
& \mathbf{U}=\left(\begin{array}{c}
\rho \\
\rho u \\
\rho v \\
\rho w \\
\rho e
\end{array}\right), \quad \overrightarrow{\mathcal{F}}=\left(\begin{array}{c}
\rho u \\
P+\rho u^{2} \\
\rho u v \\
\rho u w \\
u(P+\rho e)
\end{array}\right) \\
& \overrightarrow{\mathcal{G}}=\left(\begin{array}{c}
\rho v \\
\rho v u \\
P+\rho v^{2} \\
\rho v w \\
v(P+\rho e)
\end{array}\right), \quad \overrightarrow{\mathcal{H}}=\left(\begin{array}{c}
\rho w \\
\rho w u \\
\rho w v \\
P+\rho w^{2} \\
w(P+\rho e)
\end{array}\right)
\end{aligned}
$$

Here, $\rho, P, \mathbf{u}=(u, v, w)$ and $e$ are the fluid density, static pressure, velocity vector and total specific energy, respectively. We will consider $\gamma$-law gas, with

\footnotetext{
${ }^{1}$ Here, the model and method will be presented in the most general three-dimensional formulation. Reduction to $2 \mathrm{D}$ is straightforward.
}

equation of state $P=(\gamma-1) \rho i$, where $i$ is a specific internal energy.

The vector of source terms includes the viscous stress terms:

$$
\mathbf{S}=\left[\begin{array}{c}
0 \\
\partial_{j}\left(\mu\left(\partial_{1} u_{j}+\partial_{j} u_{1}\right)+\lambda \mathfrak{D} \delta_{1, j}\right) \\
\partial_{j}\left(\mu\left(\partial_{2} u_{j}+\partial_{j} u_{2}\right)+\lambda \mathfrak{D} \delta_{2, j}\right) \\
\partial_{j}\left(\mu\left(\partial_{3} u_{j}+\partial_{j} u_{3}\right)+\lambda \mathfrak{D} \delta_{3, j}\right) \\
0
\end{array}\right]
$$

where $\mu$ and $\lambda$ are shear and bulk viscosities, respectively. In the present study, we will neglect gravity and heat conduction terms. Furthermore, we will consider air flows - for which the following power-law dependence of $\mu$ is applicable:

$$
\begin{gathered}
\mu=\mu_{0}\left(\frac{T}{T_{0}}\right)^{\alpha_{\mu}} ; \quad \mu_{0}=1.7161 \cdot 10^{-5} \frac{\mathrm{kg}}{\mathrm{m}^{2} \cdot \mathrm{s}} \\
\alpha_{\mu}=0.76 ; \quad T_{0}=273.16 \mathrm{~K}
\end{gathered}
$$

The bulk viscosity is chosen in accordance to the Stokes' assumption, i.e. $\lambda=-\frac{2}{3} \mu$. Furthermore, $\mathfrak{D}=\partial_{j} u_{j}$ is a velocity divergence, where velocity components are numbered as $\left(u_{1}, u_{2}, u_{3}\right) \rightsquigarrow(u, v, w)$. Finally, temperature $T$ is computed from the ideal gas law equation of state, i.e., $T=\frac{P}{\rho R}$, with the specific gas constant defined as $R=\frac{R_{u}}{M o}$ $\left(R_{u}=8,314.51 \frac{\mathrm{J}}{\mathrm{kg} \cdot \mathrm{mol} \cdot \mathrm{K}}\right.$ and $\left.M o=28.97\right)$.

Non-dimensional form. Introducing the basic scales for length, density, pressure and temperature ( $\mathrm{L}, \rho_{0}$, $P_{0}$ and $\left.T_{0}\right)$, the governing equations (1), (2) and (3) can be cast in a dimensionless form, with the following non-dimensional variables: $\hat{\mathbf{x}}=\frac{\mathbf{x}}{\mathrm{L}} ; \hat{t}=\frac{t}{\mathrm{~L} \sqrt{\frac{\rho_{0}}{P_{0}}}} ; \hat{\rho}=\frac{\rho}{\rho_{0}}$; $\hat{T}=\frac{T}{T_{0}} ; \hat{P}=\frac{P}{P_{0}} ; \hat{\mathbf{u}}=\frac{\mathbf{u}}{\sqrt{P_{0} / \rho_{0}}} ; \hat{e}=\frac{e}{P_{0} / \rho_{0}}$ and $\hat{\mu}=\frac{\mu}{\rho_{0} \mathrm{~L} \sqrt{P_{0} / \rho_{0}}}$.

\section{Particle (Solid) Phase}

In the present study, the solid phase is assumed to be incompressible. Furthermore, for simplicity, we will limit ourselves to the two-dimensional configuration. Thus, the governing equations of motion for the $a^{t h}$ particle are: ${ }^{9}$

$$
\left[\begin{array}{c}
\frac{d \mathbf{V}_{a}}{d t}=\frac{\mathbf{F}_{a}}{m_{a}} \\
\frac{d \mathbf{r}_{a}}{d t}=\mathbf{V}_{a}
\end{array}\right] \text { and }\left[\begin{array}{rl}
\frac{d \Omega_{z_{a}}}{d t} & =\frac{K_{z_{a}}}{I_{z_{a}}} \\
\frac{\theta_{z_{a}}}{d t} & =\Omega_{z_{a}}
\end{array}\right]
$$

where $\mathbf{V}_{a}$ and $\mathbf{F}_{a}$ are the velocity for the center of inertia and the total force acting on the $a^{t h}$ particle of mass $m_{a}$ and radius $R_{a} ; \mathbf{r}_{a}$ and $\theta_{z_{a}}$ are the position of the center of inertia and orientation angle of the particle; $\Omega_{z_{a}}, K_{z_{a}}$ and $I_{z_{a}}$ are the angular velocity, torque and principle moment of inertia, respectively. In the case of circular particle, $I_{z_{a}}=\frac{\pi}{2} R_{a}^{4}$. The total force acting on the $a^{\text {th }}$ particle is composed of the hydrodynamical and 'collision' forces, $\mathbf{F}_{a}=\mathbf{F}_{a}^{(\mathrm{HD})}+\mathbf{F}_{a}^{(\mathrm{Col})}$. 


\section{Numerical Approach}

In this section we will outline the numerical algorithm for DNS of compressible fluid-solid particle flows.

\section{Level-Set-Based Cartesian Grid Method (LSCG)}

For description of complex dynamically evolving fluid-solid boundaries in compressible flows, we employ the Level-Set-Based $\underline{\text { Cartesian }}$ Grid (LSCG) method. Detail description of the LSCG is given in our previous studies. ${ }^{12,13,14}$ Here, we will present only a brief summary of the approach.

The physical time-space is discretized using a uniform mesh ${ }^{2}$ with constant steps $\Delta t, \Delta x, \Delta y$ and $\Delta z$. Correspondingly, the discrete nodes are denoted by indices $[\mathrm{n}, \mathrm{i}, \mathrm{j}$ and $\mathrm{k}]$. The fluid-solid boundaries in particulate flows are represented by the level set function, defined in all computational nodes as a signed distance to the boundary, $\varphi_{(\mathrm{n}, \mathrm{i}, \mathrm{j}, \mathrm{k})}$. Zero-level of this function represents an interface which separates fluid $(\varphi>0)$ and solid $(\varphi<0)$.

After each time step of the numerical solution, based on the current positions of all particles, the level set is reconstructed analytically (for circular particles) or using the PDE- and $\mathrm{WENO}_{5}$-based re-initialization technique $^{18}$ (for complex-shape particles).

Next, based on the current value of the level set function, all computational nodes are "tagged" into four different groups: i) REAL nodes (RN); ii) BOUNDARY nodes (BN); iii) GHOST nodes (GN); and $i v$ ) UNUSED nodes (for details - see (Nourgaliev et al. $\left.)^{14}\right)$. Solution in REAL nodes is obtained using the 'Characteristics-Based Conservative Finite Difference (CBCFD)' approach. Numerical solution in BNs is computed using the 'CharacteristicsBased Matching (CBM)'. GNs are populated using the $1^{\text {st }}$-order-accurate level-set-PDE-based extrapolation technique. ${ }^{18}$

\section{Treatment of Fluid Dynamics}

Time discretization. The whole numerical solution is integrated in time using the third-order accurate implicit virtual space relaxation (VSR) numerical procedure. ${ }^{16}$ The VSR procedure has been introduced in (Nourgaliev et al. $)^{13}$ and its convergence properties are studied in ref. ${ }^{16}$ In summary, the VSR is the implicit time discretization procedure, in which the third order accuracy in time is achieved using the MUSCLbased scheme. Here, we will skip the details of the VSR - referring interested readers to studies. ${ }^{13,14,16}$

Space discretization. For spatial discretization, we utilize the Characteristics-Based Conservative Finite Difference (CBCFD) approach. Detail descriptions of this method can be found in studies ${ }^{12,21}$ In summary, we use the third-order-accurate MUSCL scheme ${ }^{24}$ for

\footnotetext{
${ }^{2}$ Extension of the LSCG/CBM to adaptively refined mesh
} (AMR) technology will be presented elsewhere. extrapolation of flow variables and characteristic fluxes from the cell centers to the cell walls. The MUSCL scheme is supplemented by van Albada's limiter. ${ }^{23}$ The fluxes in characteristic space are computed using the Local Lax Friedrichs (LLF) scheme. ${ }^{12}$ The viscous diffusion terms, incorporated into the local discrete source term $\mathbf{S}_{(\mathrm{n}, \mathrm{i}, \mathrm{j}, \mathrm{k})}$, are computed using the fourthorder-accurate central differencing scheme.

\section{Characteristics-Based Matching (CBM)}

Boundary treatment. The purpose of the characteristics-based matching (CBM) is to "infuse" the desirable set of boundary conditions at BNs. ${ }^{13}$ The approach is based on the well-known one-dimensional characteristics-decomposition-based boundary treatment of compressible fluid; and makes use of the level set function to extend it to multiple dimensions. The level set allows for incorporation of the "bodyfitting" features into the framework of the Cartesian Grid method. To retain high-order accuracy near interface, the "limited MUSCL- and level-set-based procedure" has been developed, in which multidimensional polynomial reconstruction of subcell interface positions and boundary values is utilized. ${ }^{14}$ In the present paper, we will omit detail description of the CBM, referring to our previous studies ${ }^{12,13,}{ }^{14}$

\section{Particle Logics}

Implementation of the particle logics is described in details previously. ${ }^{14,15}$ In summary, particle's equations of motion (5) are discretized using the firstorder-accurate Euler backward differencing scheme. The hydrodynamical forces $\mathbf{F}_{a}^{(\mathrm{HD})}$ and torques $K_{z_{a}}$ acting on each particle are computed using the bicubic spline interpolation technique together with the level-set-based body-fitting transformation, needed to reconstruct shear and normal stresses at the particle surfaces. ${ }^{14,15}$

Collisions. For simulation of particle-particle collisions, we employ the model developed in our previous study. ${ }^{15}$ This model enables an effective simulation of both elastic and viscoelastic high-speed particle collisions. It is based on the collision model for granular gases, developed previously by Brilliantov et al., ${ }^{3}$ which incorporates existing models of impact with dissipation as well as the classical Hertz impact theory as special cases. By introducing the "effective collision properties" of particles and "effective collision force" $\mathbf{F}_{a}^{(\mathrm{Col})}$, collisions are numerically smeared over a few time steps and 1-2 computational grid nodes, allowing to eliminate stability and particle-particle overlap issues. Energy and momentum of elastic collisions are preserved, and, in the case of viscoelastic collisions, the dependence of the restitution coefficients on impact velocity is straightforwardly incorporated (see reference $^{15}$ for details of implementation and convergence study). 


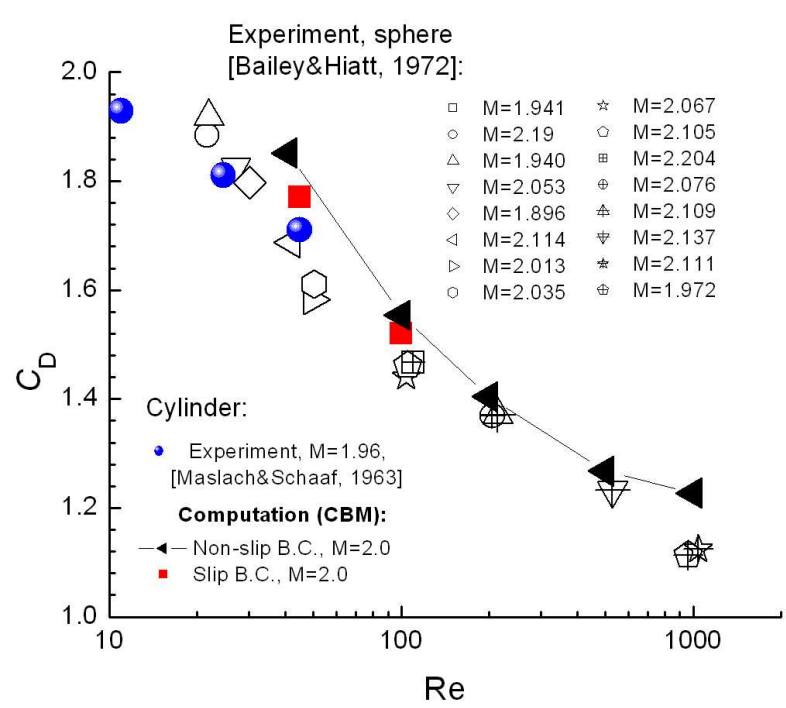

Fig. 1 The effect of $R e$ number on total drag coefficient for $M=2$.

\section{Validation}

The above-described numerical approach has been extensively validated in our previous studies ${ }^{10,12,13,14}$ for both Navier-Stokes and Euler equations, in a wide range of Mach and Reynolds numbers. For stationary boundaries, the LSCG/CBM approach produces an excellent agreement with available experimental data for total drag coefficient (refer to Fig. 1) and for dynamics of global structures of compressible flows (positions of shocks, contact discontinuities, rarefaction zones, etc. $\left.{ }^{10,12,13,14}\right)$. In terms of the total drag coefficient, the method is shown to produce the secondorder convergence. ${ }^{14}$ Performance of the LSCG/CBM for moving boundaries is examined in studies, ${ }^{13,14}$ where we have demonstrated that the LSCG/CBM approach does not suffer from the "over-/under-heating error" problems - known to be a serious issue for moving fluid-solid boundaries. ${ }^{4}$ Galilean invariance of the LSCG/CBM treatment has been demonstrated in reference, ${ }^{14}$ where we have studied convergence of the method in the case of high-speed motion of circular body.

\section{Numerical Results}

In the present section, we will investigate interaction of the shock wave with solid particles. The problem formulation for numerical tests is the following. In the computational domain of size $L_{x} \times L_{y}$, periodical in $y$-direction, we initialize cloud of circular particles, with diameter of $1 \mathrm{~mm}$. Initially, these particles are motionless. From the left, there is an incoming $M_{\mathrm{sh}}=$ 5 shock wave, defined by the following pre- and post-

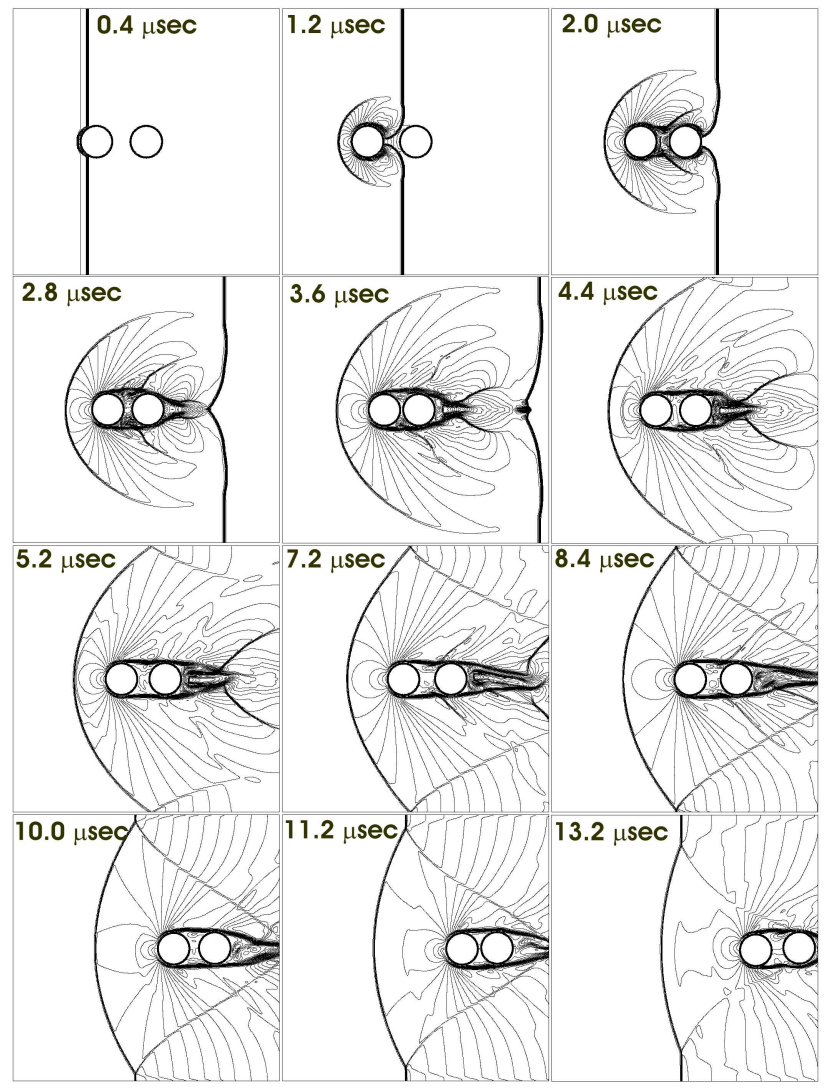

Fig. 2 Dynamics of the elastic head-to-head collision. Mach field is rendered with 30 isolines uniformly distributed in the range from 0 to 3.17 .

shock conditions:

$$
\text { Pre-shock: } \quad\left\{\begin{aligned}
P & =10^{5} \mathrm{~Pa} \\
T & =293.10344828 \mathrm{~K} \\
\mathbf{u} & =(0 ; 0) \mathrm{m} / \mathrm{s} \\
P & =2.9 \cdot 10^{6} \mathrm{~Pa} \\
T & =1,700 \mathrm{~K} \\
\mathbf{u} & =(0 ; 1,372.7092351) \mathrm{m} / \mathrm{s}
\end{aligned}\right.
$$

Driven by the impact from the transmitted shock, particles start to move to the right, colliding with each other under different impact configurations. Both elastic and viscoelastic collisions are considered. In the case of viscoelastic collision, the restitution coefficient is fixed to a value of 0.2 .

\section{Collisions of Two Isolated Particles}

First, we will study interaction of shock wave with two isolated particles. The focus is placed on the demonstration and testing of the collision model.

\section{Head-to-Head Collision}

As a first example, we will consider a head-tohead collision. The size of the computational domain is $(8 \times 8) \mathrm{mm}$. Initial positions of particles are $\mathbf{r}_{1}=(2.5 ; 4.0) \mathrm{mm}$ and $\mathbf{r}_{2}=(4.0 ; 4.0) \mathrm{mm}$. To speedup the dynamics, we will consider artificially light particles with an effective density $\rho_{\mathrm{p}}=100 \frac{\mathrm{kg}}{\mathrm{m}^{3}}$ ("empty shells"). Initial position of the shock is $1 \mathrm{~mm}$ to the left of the center of particle "1". Simulations are 

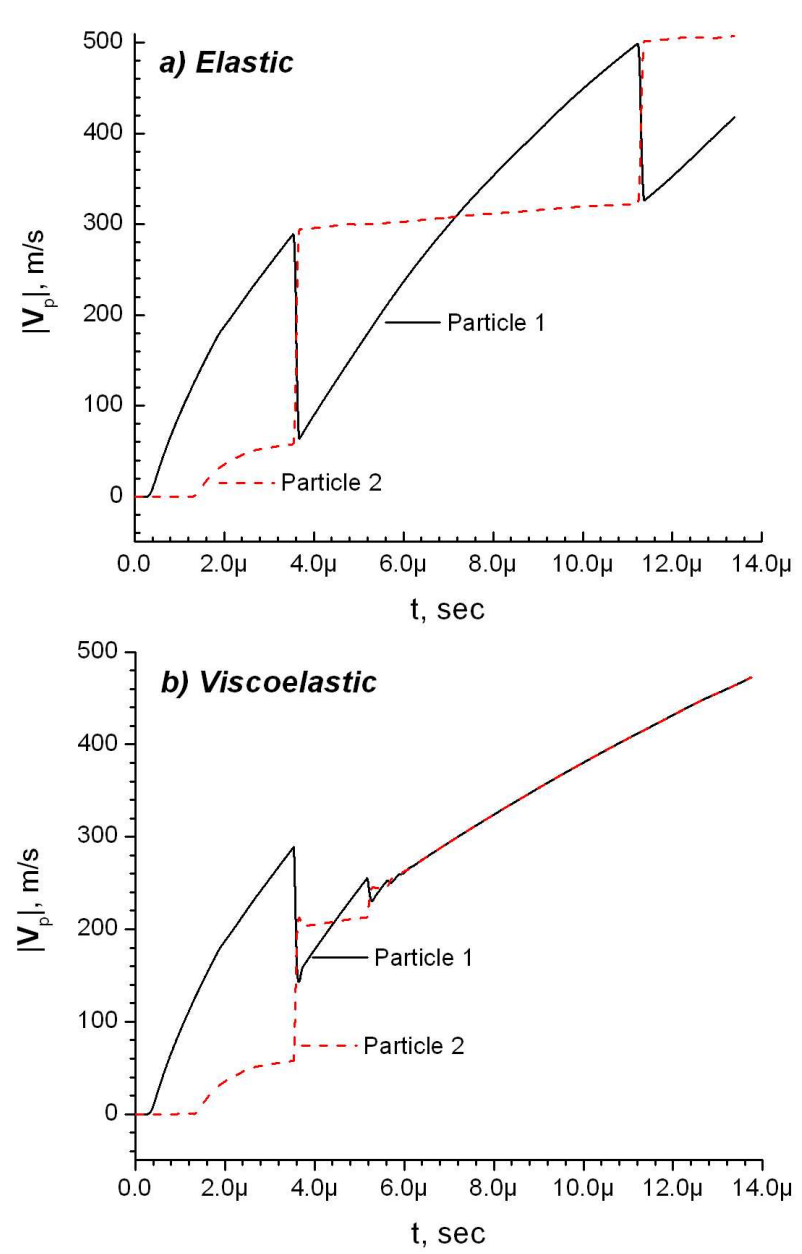

Fig. 3 Dynamics of particle's velocities (absolute value) for simulation of a) elastic and b) viscoelastic head-to-head collision.

performed with grid resolution of 40 computational nodes per particle's diameter, and time step of $\Delta t=5$ nsec. This corresponds to the CFL number of up to $\sigma_{t}=0.602$.

Dynamics of collisions. History of the Mach field and particle's positions for elastic collision are shown in Fig. 2. Within the first $13 \mu$ secs of the simulations, two collisions took place. As seen from Fig. 3a, the first particle, accelerated to the velocity of approximately $300 \mathrm{~m} / \mathrm{s}$, hits the second particle at the moment of approximately $t_{c}=3.5 \mu \mathrm{secs}$, with impact velocity of $260 \mathrm{~m} / \mathrm{s}$. Due to the elastic collision, the particles exchange momentum - i.e., the first particle decelerates to the velocity of approximately $60 \mathrm{~m} / \mathrm{s}$, while the second particle accelerates to $\approx 300 \mathrm{~m} / \mathrm{s}$. Collision is numerically smeared over approximately 30 time steps, see Figs. 4a-d. Figs. 4a and b depict the dynamics of the specific collision force ${ }^{3} F_{N}^{(\mathrm{Col})}$ and integral of the total impulse $I^{(\mathrm{Col})}$ due to collision. The history of the

\footnotetext{
${ }^{3}$ Since we are considering $2 \mathrm{D}$ formulation, the specific force and impulse are defined as force and impulse per unit length in $z$-direction.
}
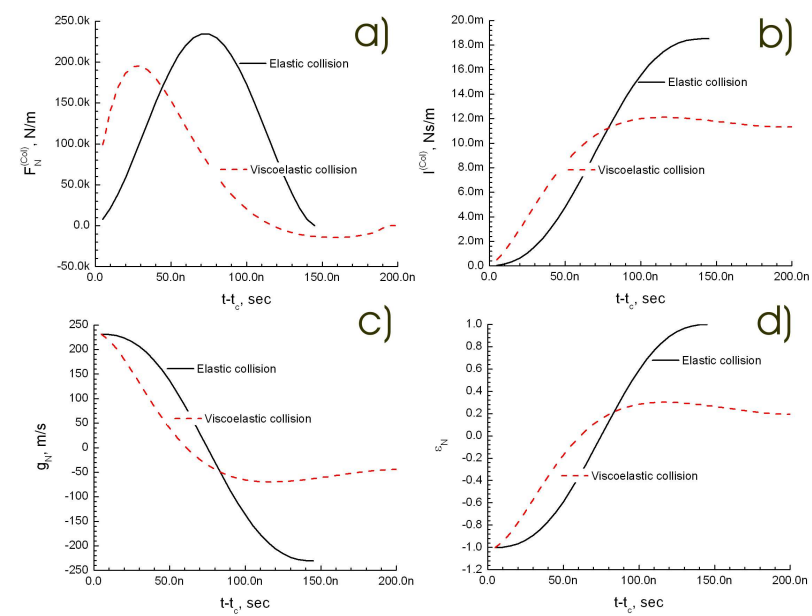

Fig. 4 "Numerical smearing" of particle-particle collisions over a few time steps.

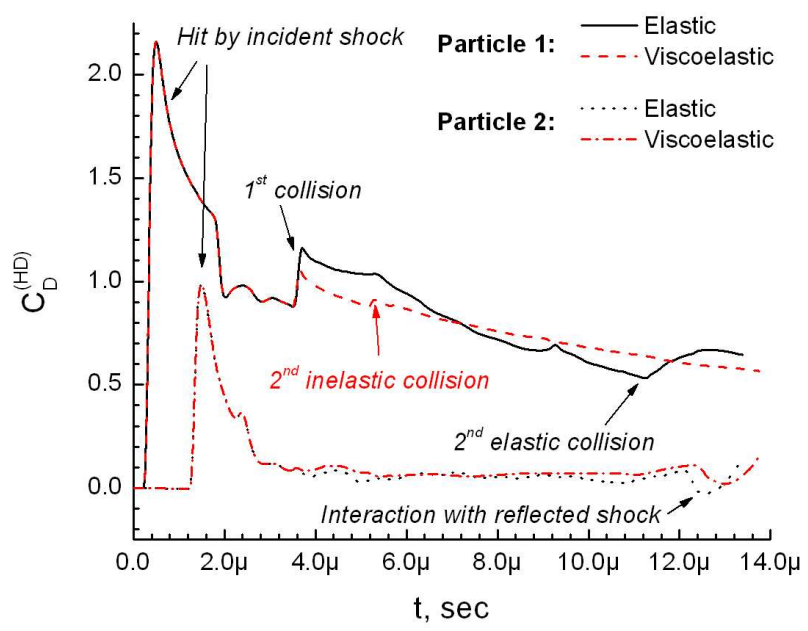

Fig. 5 On comparison of the total drag coefficient for simulation of elastic and viscoelastic head-tohead collision.

relative velocity is shown in Fig. 4c. Fig. 4d demonstrates how the restitution coefficient $\varepsilon_{N}$, defined as a ratio of relative velocities before and after the impact, changes in time. At the end of the collision $\varepsilon_{N}=1$, which indicates that it is a perfectly elastic collision. The total drag coefficient acting on both particles is shown in Fig. 5. As it can be seen, each collision causes an increase of the total drag acting on the first particle, due to sudden deceleration and correspondent increase of pressure in front of the particle. The effect of the collision on the second particle is minor, due to the screening effect of the first particle. Next, from Fig. 5, one can observe interaction with the reflected shock at approximately $13^{\text {th }} \mu$ sec of the simulation. This reflected shock comes from the neighbor couple of particles (we utilized a periodical boundary conditions in $y$-direction). The reflected shock, which can be seen in Fig. 2 starting from $t=4.4 \mu \mathrm{sec}$, hits the second particle and, then - dissipates. Thus, its effect on the first particle is negligible. 


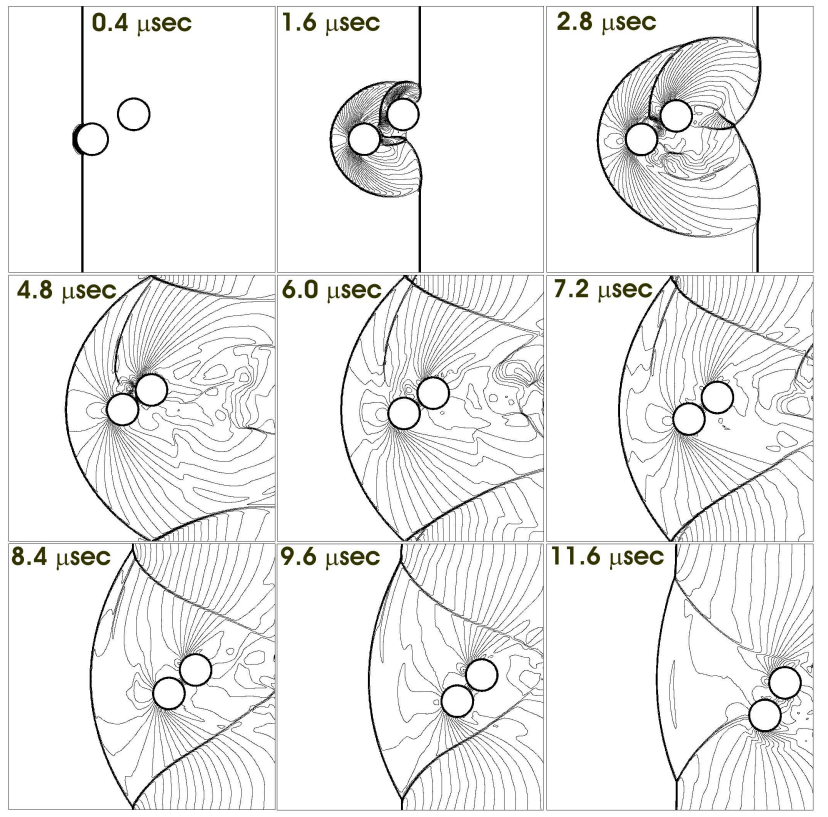

Fig. 6 Dynamics of the viscoelastic offset collision. Pressure field is rendered with 50 isolines uniformly distributed in the range from 0 to 177.5 bars.

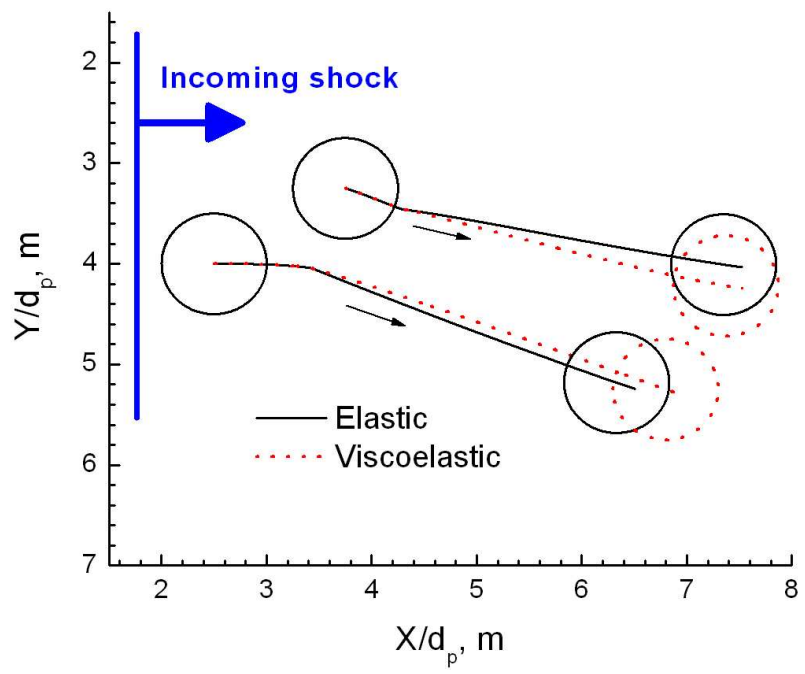

Fig. 7 Particle's trajectories for simulation of elastic and viscoelastic offset collision.

Inelastic collision. In the case of the viscoelastic collision, the restitution coefficient was prescribed to a value of $\varepsilon_{N}=0.2$. In this case, the collision force is a function of the collision deformation $\varsigma$ and its rate $\dot{\varsigma}$, i.e. $F_{N}^{(\mathrm{col})}=C \varsigma^{1 / 2}(\varsigma+A \dot{\varsigma})$, where $C$ and $A$ are the coefficients of elasticity and viscoelasticity, correspondingly (for details refer to ref. ${ }^{15}$ ). As a result, the collision force is asymmetrical in collision time, Fig. 4a. Furthermore, due to $\dot{\varsigma}$ term, for a short time at the end of the collision, the collision force is attractive. The duration of the inelastic collision is increased, relative to the elastic one. The restitution coefficient of $\varepsilon_{N}=0.2$ is perfectly recovered. Fig. $3 \mathrm{~b}$ shows the dynamics of particle's velocities, from which signifi-
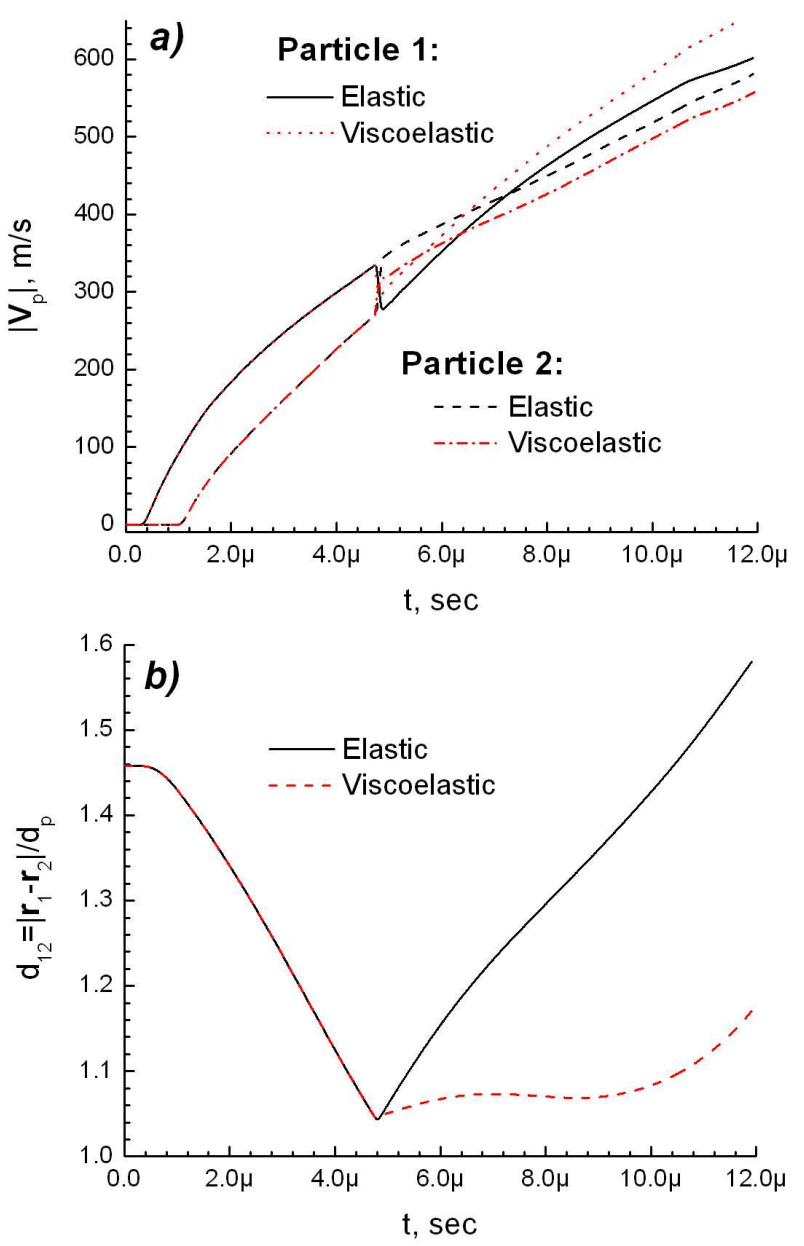

Fig. 8 Dynamics of a) particle's velocities (absolute value) and b) distance between particles for simulation of elastic and viscoelastic offset collision.

cant reduction of $\left|\mathbf{V}_{p}\right|$ due inelastic collision can be observed. Furthermore, due to smaller value of the relative velocity after the first collision, the second collision happens earlier than in the case of the elastic collision (Fig. 3a vs. b). After the second viscoelastic collision, two particles "cluster" into one "combined body", moving with essentially the same velocity. It is instructive to note that while at the beginning this velocity is higher than the velocity of the first particle in the case of the elastic collision, it becomes lower very soon, due to the increased inertia of the "combined body". As a result, starting from approximately $7^{t h}$ $\mu$ sec, the total drag of the inelastic simulation exceeds the one of the elastic simulation, Fig. 5.

\section{Offset Collision}

In the next example, we will consider offset collision of two isolated particles. Problem formulation is essentially the same as for the head-to-head collision, except that the initial position of the center of the second particle is $\mathbf{r}_{2}=(3.75 ; 3.25) \mathrm{mm}$.

Dynamics of the pressure field in the case of viscoelastic offset collision is shown in Fig. 6. In this 
case, there is only one collision, which occurred at the simulation time of $t_{\mathrm{c}}=4.72 \mu \mathrm{sec}$. This collision causes deflection of particle's trajectories, depicted in Fig. 7. Notably, similarly to the case of the head-to-head collision, the viscoelasticity tends to "cluster" particles - i.e., both the deflection angle and distance between particles is less compared to those in the case of the elastic collision, see Figs. 7 and 8. Furthermore, while in the case of the elastic collision the relative velocity of particles increased, in the case of the viscoelastic collision, it remains the same (see Fig. 8a).

The calculation of two-particle collisions demonstrate the importance of inelasticity - which tends to "cluster" particles, in difference to the elastic collisions - resulting in less significant dispersal of particles. Apparently, this effect should impact on the global behavior of dense multi-particle disperse system, which will be considered next.

\section{Interaction of the $\mathrm{M}=5$ Shock Wave with Isolated Clusters of Particles}

Problem formulation. As our final example, we will consider 5-layer-cloud of solid particles, arranged in isolated cluster formations, each composed of 12 particles as shown in Fig. 9a. The size of the computational domain, periodical in $y$-direction, is $(24 \times 8) \mathrm{mm}$. The initial mean volume fraction of the cloud is 0.18 . Initial particle's positions are $\mathbf{r}_{1}=(9 ; 4), \mathbf{r}_{2}=(10.25 ; 3.25)$, $\mathbf{r}_{3}=(10.25 ; 4.75), \mathbf{r}_{4}=(11.5 ; 2.5), \mathbf{r}_{5}=(11.5 ; 5.5)$, $\mathbf{r}_{6}=(11.5 ; 4), \mathbf{r}_{7}=(13 ; 4), \mathbf{r}_{8}=(13 ; 2.5), \mathbf{r}_{9}=$ $(13 ; 5.4), \mathbf{r}_{10}=(14.5 ; 4), \mathbf{r}_{11}=(14.5 ; 2.5), \mathbf{r}_{12}=$ $(14.5 ; 5.5) \mathrm{mm}$. With this setup, the initial mean volume fraction of each cluster is 0.48 . The density of particles is $\rho_{\mathrm{p}}=2,000 \frac{\mathrm{kg}}{\mathrm{m}^{3}}$ (ceramic dust).

Initial position of the shock is $1 \mathrm{~mm}$ to the left of the center of the first particle. Simulations are performed using two different grids with resolution of 10 and 40 computational nodes per particle's diameter (approximately $2 \cdot 10^{4}$ and $3 \cdot 10^{5}$ nodes in total, respectively) and time steps of $\Delta t=20$ and 5 nsec, which corresponds to the CFL number of up to $\sigma_{t}=0.744$.

Dynamics of interaction. Figs. 10 and 11 show the history of the incident shock - particle cloud interaction, at the very beginning of the simulation. The incident shock, moving with approximately $1,700 \mathrm{~m} / \mathrm{s}$ hits the cluster. This generates the transmitted and reflected shocks. The transmitted shock passes through the particle cloud within the first $5 \mu$ sec and leaves the computational domain in $10 \mu \mathrm{sec}$. The reflected shock, originated as a combination of the bow shocks at the leading edge particles (see Fig. 11), moves slowly to the left, with velocity of approximately $-200 \mathrm{~m} / \mathrm{s}$, and leaves the computational domain in approximately $45 \mu \mathrm{sec}$.
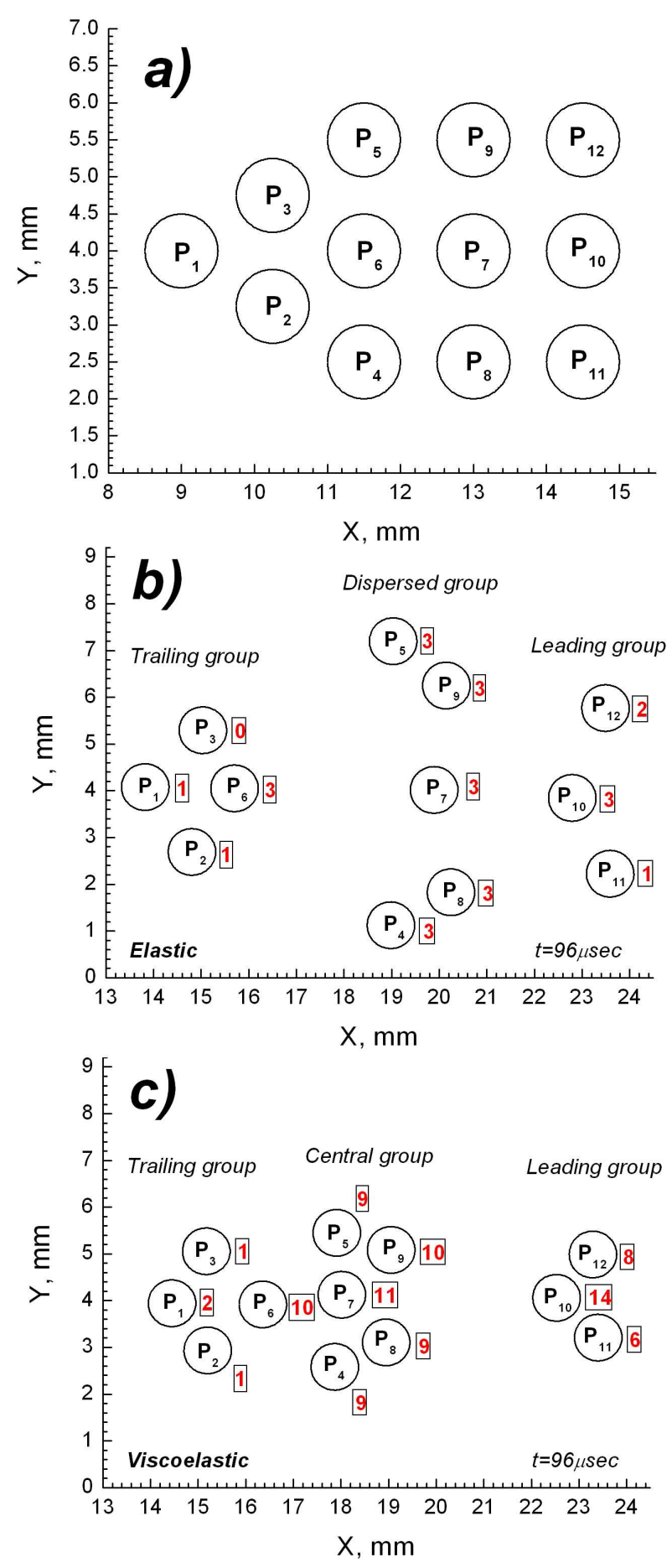

Fig. 9 a) Initial configuration of the isolated cluster of particles. b) Final configuration of particles in the case of elastic collisions. c) Final configuration of particles in the case of viscoelastic collisions. Grid resolution is 10 nodes per diameter. The total number of collisions experienced by particle is shown in boxes.

Fig. 12 depicts the spatial distributions of the Mach 


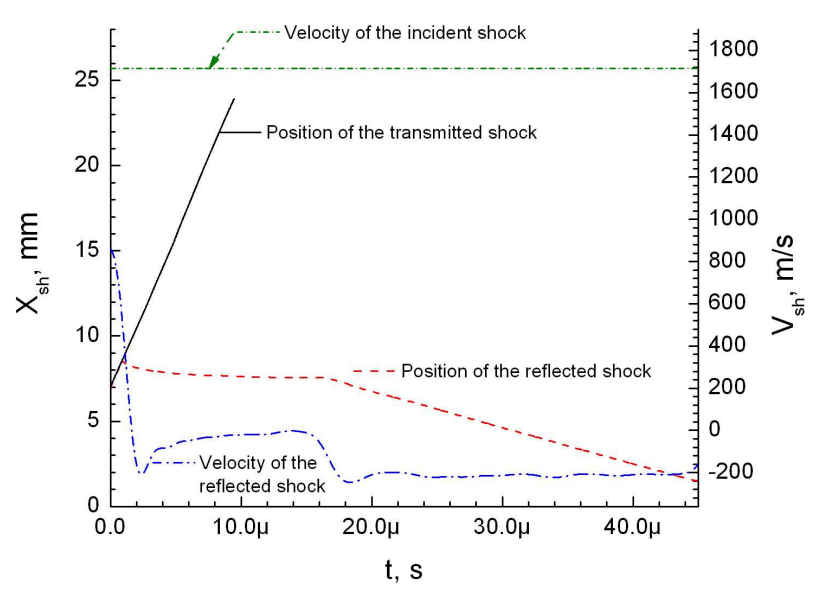

Fig. 10 Dynamics of the position and velocity of the transmitted and reflected shock.

number and pressure, averaged as

$$
\underline{\Psi}(x)=\frac{\int_{A^{\text {gas })}(x)} \Psi(x, y) d A(x)}{A^{\text {(gas) }}(x)}
$$

where $\Psi=M$ or $P$; and $A^{\text {(gas) }}(x)$ is the total crosssection area occupied by gas at the position $x$; for time $t=32 \mu \mathrm{sec}$. As it can be seen, in front of the cluster, there is a layer of the gas, compressed to approximately 120 bars, which corresponds to approximately 100 bars pressure difference across the particle cloud. Driven by this pressure difference, the cloud relocates to the right with the velocity increasing from 0 to approximately $150 \mathrm{~m} / \mathrm{s}$ at the end of the simulation. Fig. 13 shows the history of the particle's center of inertia $\mathbf{R}_{\text {c.i. }}=\frac{\sum_{a}^{N_{p}} m_{a} \mathbf{r}_{a}}{\sum_{a}^{N_{p}} m_{a}}=\left(x_{\text {c.i. }} ; y_{\text {c.i. }}\right)$, plotted for both elastic and viscoelastic simulations. Computing the second derivative of $\mathbf{R}_{\text {c.i. }}$ in time, one can reconstruct the mean drag coefficient for a cluster, $\underline{C}_{D}$, shown in Fig. 14. The mean drag coefficient varies in the range from 0.4 to 0.53 , which indicates that particles in the cluster are subjected to the subsonic flow ${ }^{4}$. Indeed, as it can be seen in Fig. 12, the major part of the flow in the particle cloud is subsonic. In addition, in Fig. 14, we plotted $\underline{C}_{D}$ computed as an average over all particles, i.e. $\underline{C}_{D}=\frac{\sum_{a}^{N_{p}} C_{D_{a}}}{N_{p}}$, where $C_{D_{a}}$ and $N_{p}$ are the total drag coefficient for the $a^{\text {th }}$ particle and the total number of particles, respectively. Consistency of different ways to compute the mean drag coefficient is apparent.

Using $\mathbf{R}_{\text {c.i. }}$ one can define the characteristic length scales of the particle cloud as

$$
\underline{L}=\frac{\sum_{a}^{N_{p}}\left|\mathbf{R}_{\mathrm{c} . \mathrm{i} .}-\mathbf{r}_{a}\right|}{N_{p}} ; \quad \underline{L}_{x}=\frac{\sum_{a}^{N_{p}}\left|x_{\mathrm{c.i.}}-x_{a}\right|}{N_{p}}
$$

\footnotetext{
${ }^{4}$ It is instructive to note that the drag coefficient in the case
} of the supersonic flow is above 1 , see Fig. 1 and ref. ${ }^{10}$

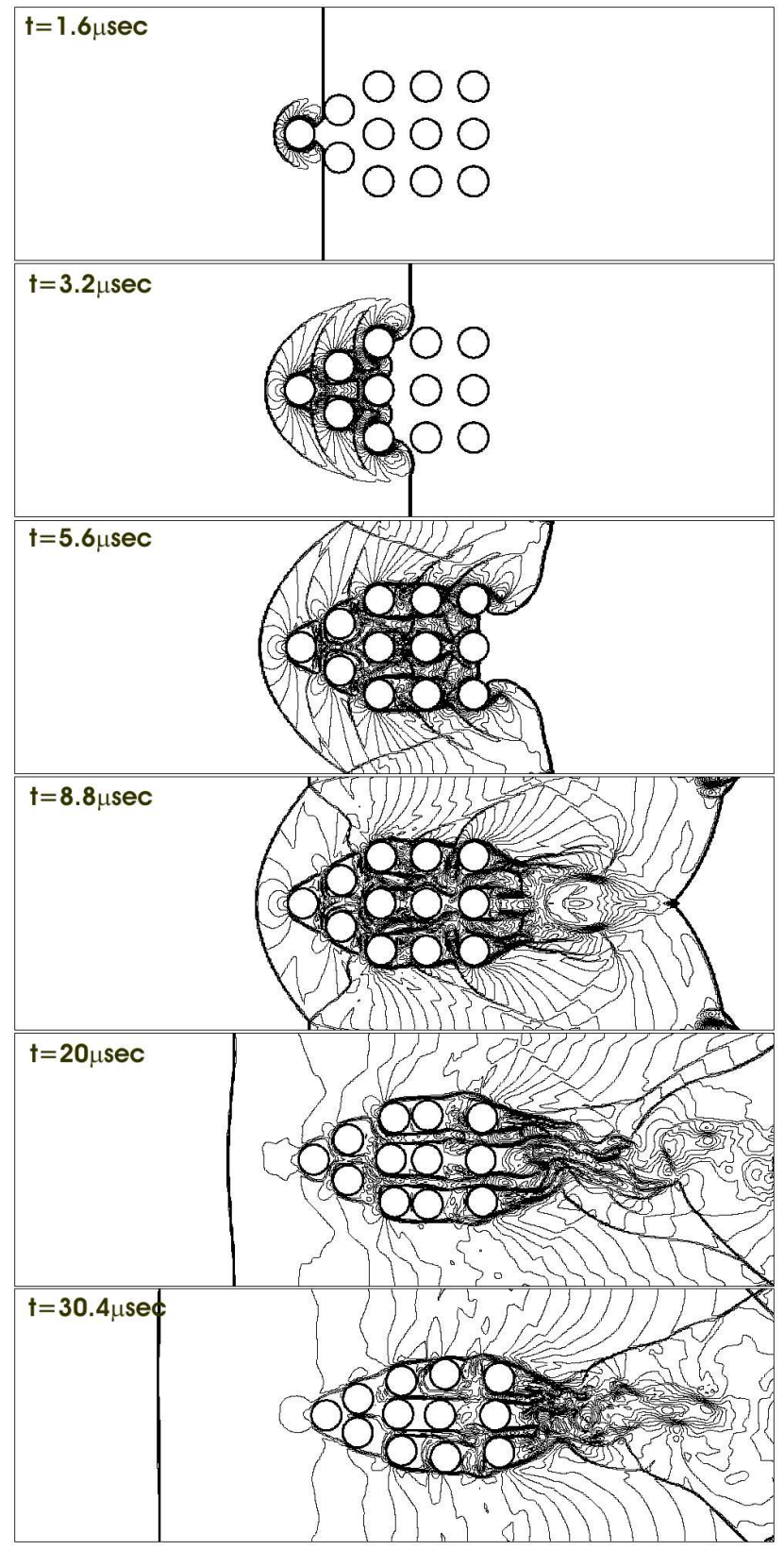

Fig. 11 Dynamics of the Mach number at the "compression" stage. Mach field is rendered with 30 isolines uniformly distributed in the range from 0 to 4.6. Grid resolution is 40 nodes per diameter.

$$
\text { and } \quad \underline{L}_{y}=\frac{\sum_{a}^{N_{p}}\left|y_{\text {c.i. }}-y_{a}\right|}{N_{p}}
$$

These length scales are plotted in Fig. 15 for elastic and viscoelastic simulations.

Dispersal in elastic simulation. In the case of elastic simulation, one can distinguish three stages of the particle cloud dynamics. At the first stage (up to approximately $18 \mu \mathrm{sec}$ ), shown in Fig. 11, the cluster is compressed in both $x$ and $y$ directions. This can also be observed from the dynamics of $\underline{L}_{x}$ and $\underline{L}_{y}$, Fig. 15 . The second stage is characterized by the increase in 


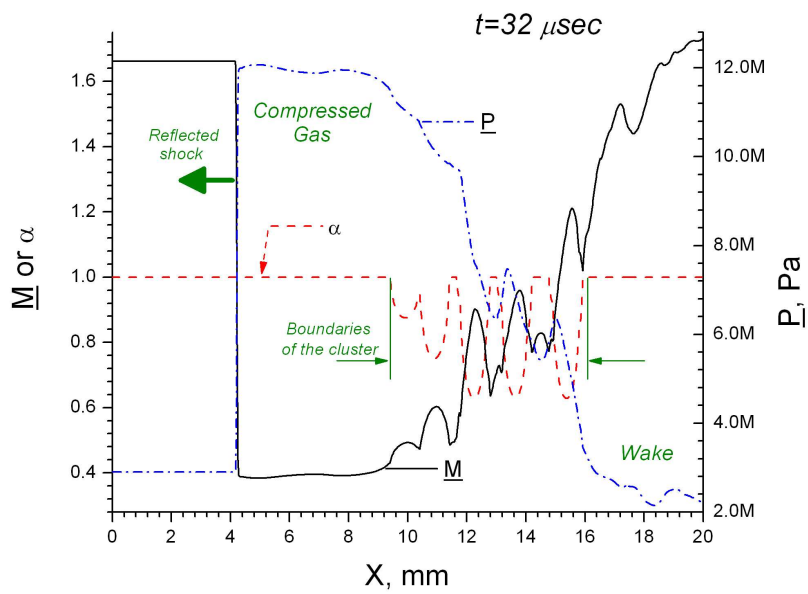

Fig. 12 Distribution of the averaged Mach number $\underline{M}$, pressure $\underline{P}$ and gas volume fraction $\alpha$ for time $t=32 \mu$ sec. Grid resolution is $\mathbf{4 0}$ nodes per diameter.

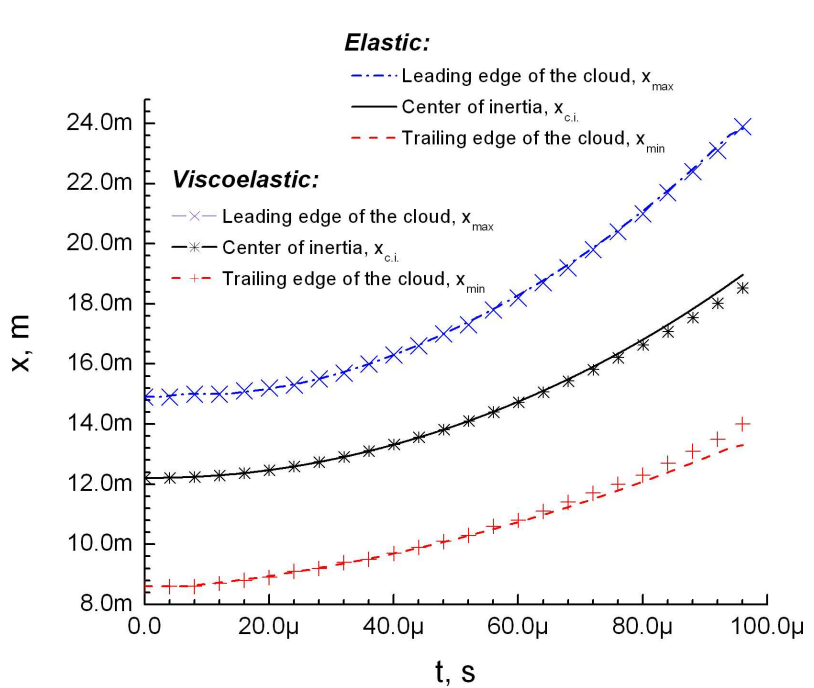

Fig. 13 Positions of the cluster's center of inertia and boundaries of the particle cloud as functions of time. Grid resolution is $\mathbf{1 0}$ nodes per diameter.

$\underline{L}_{x}$ - 'elongation', accompanied by the decrease of the scale $\underline{L}_{y}$. At the final stage of the elastic simulation, there is a drastic increase of both $\underline{L}_{y}$ and $\underline{L}$, which corresponds to the lateral dispersal of solid material, as shown in Fig. 16. Fig. 9b shows the final configuration of the particle cloud together with the total number of collisions experienced by each particle (written in boxes). There are three distinct groups of particles: the 'leading', the 'dispersed' and the 'trailing'. The 'trailing' group is subjected to the fewest number of collisions. As a result - the particles of this group retain the original 'formation'. Next, as it can be seen, the majority of collisions took place between particles of the 'dispersed' group - i.e. the particles in the center of the cluster are subjected to the maximum "particle pressure", which leads to the significant lateral dispersal. Particles of the 'leading' group are stripped

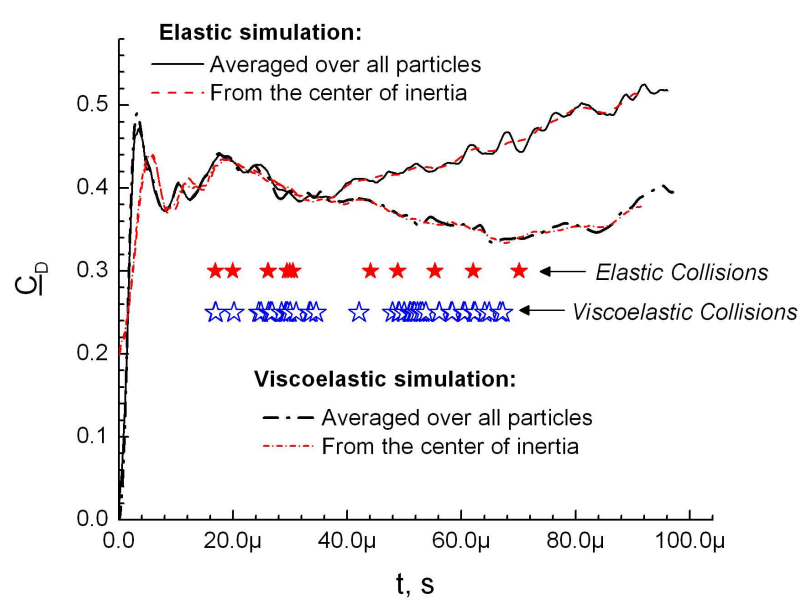

Fig. 14 Dynamics of the mean total drag coefficient for a cluster. Grid resolution is 10 nodes per diameter.

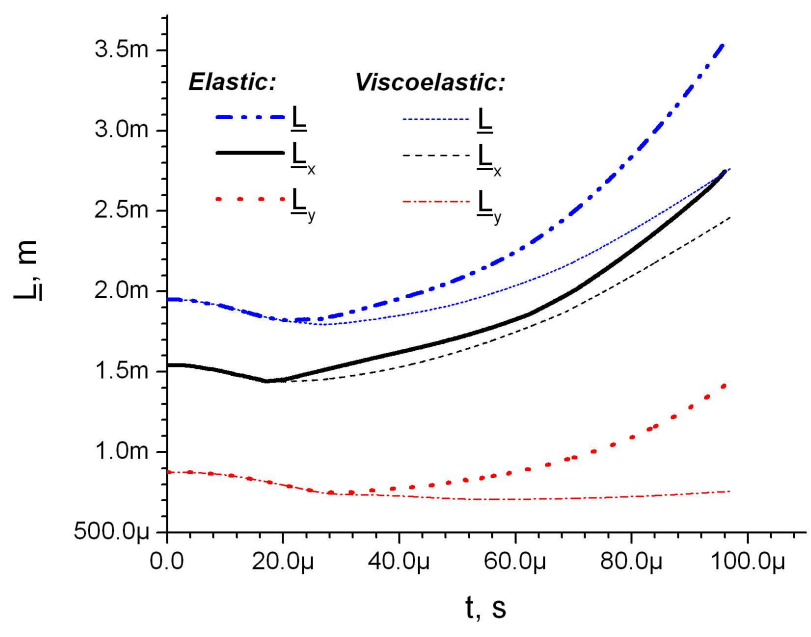

Fig. 15 Dynamics of the characteristic scales of the cluster for the case of elastic and viscoelastic collisions. Grid resolution is $\mathbf{1 0}$ nodes per diameter.

away from the cluster at the 'elongation' stage. Particle ' 10 ', squeezed between particles ' 11 ' and ' 12 ', is subjected to three elastic 'bounceback' collisions with them, which produced some lateral dispersal in this group. It is instructive to note that, because of the natural instability of the flow (formation of the 'von Karman vortex street', visible in Figs. 11, 16 and 17), there is an asymmetry in particle collisions.

Viscoelastic simulation. Dynamics of the particle cloud dispersal is quite different under viscoelastic collisions. First, there is no lateral spreading of the material, i.e. $\underline{L}_{y} \approx$ const after $t=20 \mu \mathrm{sec}$, see Fig. 15. The total number of collisions, shown as open stars in Fig. 14, is significantly higher, than in the case of elastic collisions (45 vs. 13). This is because the collisions are dissipative and the particles do not run away from each other due to the 'particle pressure', but rather they prefer to 'cluster'. This can also be seen in Figs. 9b,c, 16 and 17. Similarly to the 


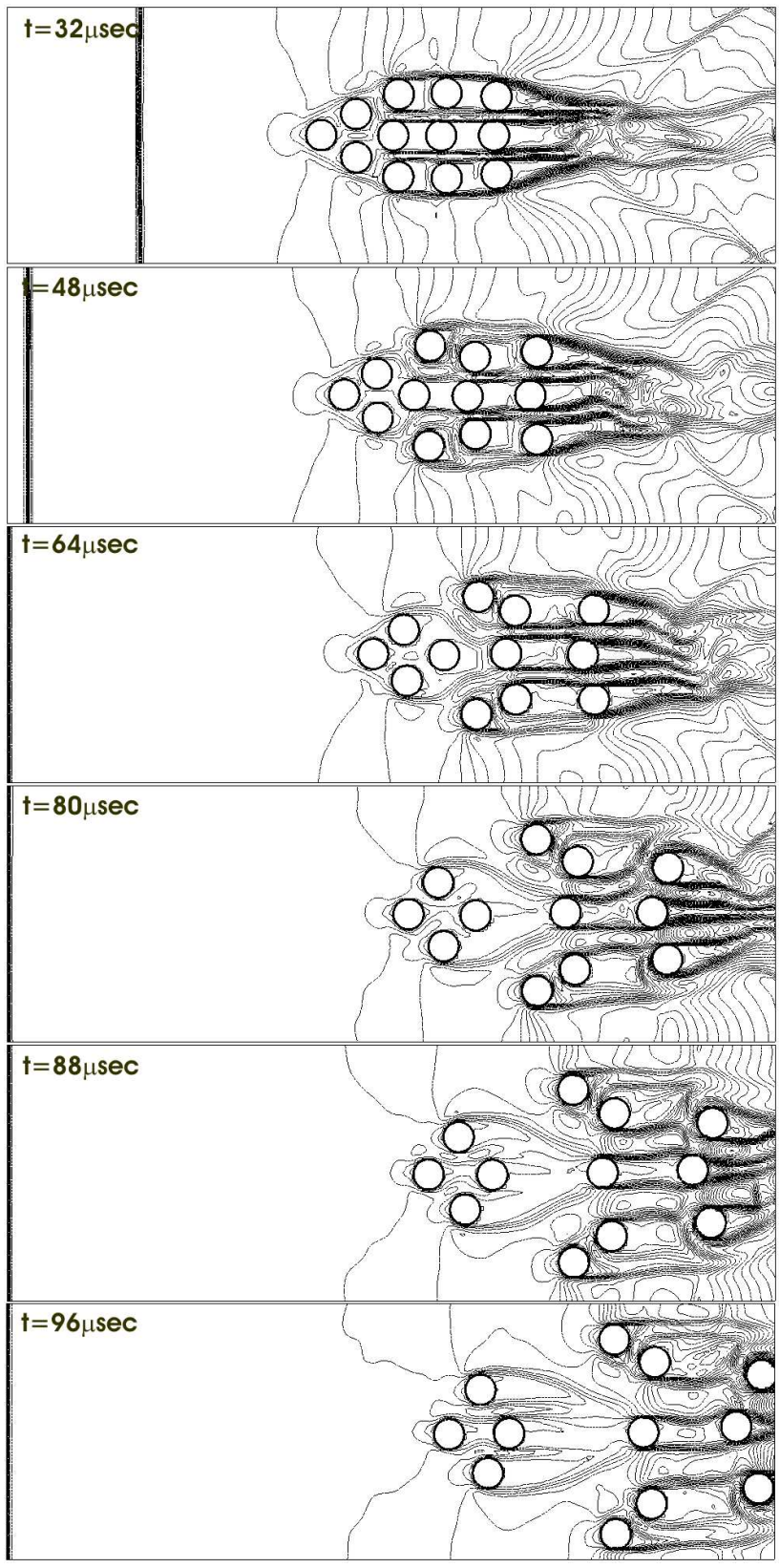

Fig. 16 Dynamics of the Mach number at the "elongation \& dispersion" stages. Elastic collisions. Mach field is rendered with 30 isolines uniformly distributed in the range from 0 to 4.6. Grid resolution is 10 nodes per diameter.

elastic simulation, particles of the "trailing" group experience the fewest number of collisions; the particles of the "central" group are under the highest "particle pressure' conditions, and, finally, particles of the "leading" group are stripped away from the rest of the cloud. Comparing dynamics of the center of inertia for particle cloud and the 'trailing' and 'leading' edges of the cloud (Fig. 13), one can see that, in the case of the viscoelastic simulation, the cloud moves slightly slower and it is also slightly more compact in the $x$ direction. The mean total $\operatorname{drag} \underline{C}_{D}$, acting on particle cloud, is significantly lower in the case of the viscoelas-

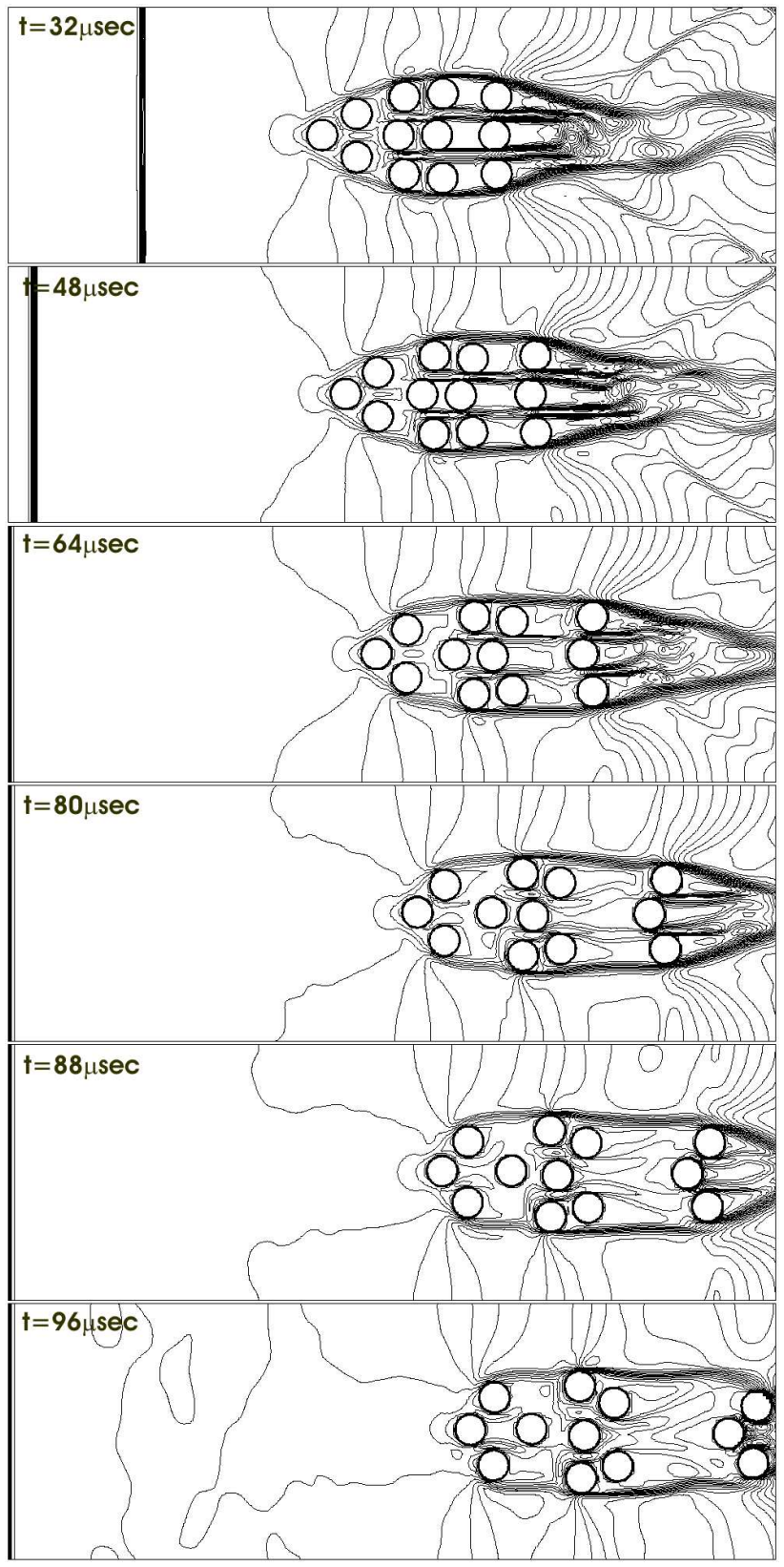

Fig. 17 Dynamics of the Mach number at the "elongation \& dispersion" stages. Viscoelastic collisions. Mach field is rendered with 30 isolines uniformly distributed in the range from 0 to 4.6. Grid resolution is $\mathbf{1 0}$ nodes per diameter.

tic collisions, which can be explained by the absence of lateral spreading of the particle cloud and, as a result, the smaller projection area of the cloud exposed to the incoming flow.

\section{Concluding Remarks}

These first-of-a-kind direct numerical simulations of shock-induced dispersal of dense solid particle clouds demonstrate the complexity and richness of compressible multiphase flow physics, perhaps even at levels higher than could be anticipated. Evident is also made the power of such simulations to reveal internal intricate details that are 
prohibitively inaccessible to experimental probing of such flows.

The present numerical simulations were made possible by a novel computational approach developed and described in the paper, namely the Level-Set-based Cartesian Grid Characteristics-Based-Matching (LSCG/CBM) method. The method is essential for accurate capturing of particle-to-particle long-range interactions by a high-order accurate treatment of compressible flow fields, with shock waves and contact discontinuities emanating from moving objects (particles). Specifically, we found a drastic effect of particle-to-particle interactions on the effective drag, which is responsible for the motion, dispersal, deformation and breakup of particles in supersonic flow.

More broadly, the simulation results presented in the paper demonstrated that behavior of an individual particle in the particle cloud as well as collective behavior of the whole cloud depends, to a significant extent, on the nature of short-range, particle-to-particle interactions, i.e. collisions. Toward this end, we employed a novel method for the treatment of elastic and viscoelastic collisions. ${ }^{15}$ In itself, the collision modeling presents a significant computational challenge because of the multi-body nature of the interactions, including the fluid medium. We approach the task through a multi-scale treatment, with the objective to obtain a robust, weak solution of the particle-to-particle collision (instantaneous, local, sub-grid-scale interaction) on the grid- and time-scale used to solve the continuum problem. In a truly multi-physics multi-scale problem, where scale-to-scale interactions do matter, sub-grid-scale phenomena may greatly affect and even alter the global behavior. Interestingly, the present simulations even with a limited number of particles (and collisions) readily demonstrate that dissipative nature of viscoelastic collisions gives rise to particle clustering. In the near future, we expect to see more clearly formations of organizing patterns in simulations with larger numbers of particles.

Finally, we note that the approach and capability developed in this work, and the understanding of collective behavior and self-organization to be derived from DNS of compressible multiphase flows are instrumental to the development of effective-field models for such flows. The grand challenge is to find a way to effectively represent the non-local nature and effects of both short-range and long-range particle-to-particle interactions in a local (differential) model.

\section{References}

${ }^{1}$ Aidun, C. K., Lu, Y., and Ding, E., Direct Analysis of Particulate Suspensions with Inertia using the Discrete Boltzmann Equation, Journal of Fluid Mechanics, 373, pp.287-311, 1998.

${ }^{2}$ Boiko, V.M., Kiselev, V.P., Kiselev, S.P., Papyrin, A.N., Poplavsky, S.V., and Fomin, V.M., Shock Wave Interaction with a Cloud of Particles, Shock Waves, 7, pp.275-285, 1997.

${ }^{3}$ Brilliantov, N.V., Spahn, F., Hertzsch, J.-M., and Poschel, T., Model for Collisions in Granular Gases, Physical Review E, 53(5), pp.5382-5392, May 1996.

${ }^{4}$ Fedkiw, R.P., Marquina, A., and Merriman, B., An Isobaric Fix for the Overheating Problem in Multimaterial Compressible Flows, Journal of Computational Physics, 148, 545-578 (1999).

${ }^{5}$ Fedkiw, R.P., Aslam, T., Merriman, B., and Osher, S., A Non-oscillatory Eulerian Approach to Interfaces in Multimate- rial Flows (the Ghost Fluid Method), Journal of Computational Physics, 152, 457-492 (1999).

${ }^{6}$ Glowinski, R., Pan, T.-W., Hesla, T. I., and Joseph, D. D., A Distributed Lagrange Multiplier/Fictitious Domain Method for Particulate Flows, International Journal of Multiphase Flow, 25, pp.755-794, 1999

${ }^{7}$ Johnson, A.A., and Tezduaer, T.E., 3D Simulation of FluidParticle Interactions with the Number of Particles Reaching 100, Comput. Methods. Appl. Mech. Engrg., 145, pp.301-321, 1997.

${ }^{8}$ Ladd, A.J.C., and Verberg, R., Lattice-Boltzmann Simulations of Particle-Fluid Suspensions, Journal of Statistical Physics, 104, pp.1191-1251, 2001.

${ }^{9}$ Landau, L.D., and Lifschitz, E.M., Theoretical Physics, v.1: Mechanics, Nauka, Moscow, 1988 (Fourth Edition).

${ }^{10} \mathrm{Li}$, G.J., Nourgaliev, R.R., Dinh, T.N., and Theofanous, T.G., Particle-to-Particle Long-Range Interaction and Drag in Supersonic Flows, AIAA 2004-1064, 42nd AIAA Aerospace Sciences Meeting and Exhibit, January 5-8, 2004, Reno, NV, USA.

${ }^{11}$ Mauri, B., Direct Simulations of 2D Fluid-Particle Flows in Biperiodic Domains, Journal of Computational Physics, 156, 325-351, 1999.

${ }^{12}$ Nourgaliev, R.R., Dinh, T.N., Suschikh, S.Yu., Yuen, W.W., and Theofanous, T.G., The Characteristics-Based Matching Method for Compressible Flow in Complex Geometries, AIAA 2003-0247, 41st AIAA Aerospace Sciences Meeting and Exhibit, January 6-9, 2003, Reno, NV, USA.

${ }^{13}$ Nourgaliev, R.R., Dinh, T.N., and Theofanous, T.G., The 'Characteristics-Based Matching' (CBM) Method for Compressible Flow with Moving Boundaries and Interfaces, ASME Journal of Fluids Engineering (in press), 2003.

${ }^{14}$ Nourgaliev, R.R., Dinh, T.N., and Theofanous, T.G., On Capturing of Interfaces in Multimaterial Compressible Flows using a Level-Set-Based Cartesian Grid Method. Multiphase Compressible Fluid-Solid (Particulate) Flows, CRSS Research Report 05/03-1, 53p., May 23, 2003.

${ }^{15}$ Nourgaliev, R.R., Dinh, T.N., and Theofanous, T.G., On Modeling of Elastic and Viscoelastic Collisions in Direct Numerical Simulation (DNS) of High-Speed Multiphase Compressible Fluid-Particle Flows. Shock-Induced Dispersal of Solid Material, CRSS Research Report 11/04-1, November 10, 2003.

${ }^{16}$ Nourgaliev, R.R., Liou, M.-S., Dinh, T.N., and Theofanous, T.G., On High-Order-Accurate Implicit Time Discretization using Virtual Space Relaxation (VSR) for Computation of Compressible Flows, Manuscript under preparation, 2003.

${ }^{17}$ Osher, S., and Sethian, J.A., Fronts Propagating with Curvature-Dependent Speed: Algorithms Based on HamiltonJacobi Formulations, Journal of Computational Physics, 79, $12-49,1988$.

${ }^{18}$ Peng, D.P., Merriman, B., Osher, S., Zhao, H., and Kang, M., A PDE-Based Fast Local Level Set Method, Journal of Computational Physics, 155, pp.410-438, 1999.

${ }^{19}$ Pember, R.B., Bell, J.B., Colella, P., Crutchfield, W.Y., and Welcome, M.L., An Adaptive Cartesian Grid Method for Unsteady Compressible Flow in Irregular Regions, Journal of Computational Physics, 120, pp.278-304, 1995.

${ }^{20}$ Rogue, X., Rodriguez, G., Haas, J. F., and Saurel, R., Experimental and Numerical Investigation of the Shock-Induced Fluidization of a Particles Bed, Shock Waves, 8, 29-45, 1998.

${ }^{21} \mathrm{Shu}$, C.-W., Essentially Non-Oscillatory and Weighted Essentially Non-Oscillatory Schemes for Hyperbolic Conservation Laws, NASA/CR-97-206253, ICASE Report No. 97-65.

${ }^{22}$ Zhang, F., Frost, D. L., Thibault, P. A., and Murray, S.B., Explosive Dispersal of Solid Particles, Shock Waves, 10, pp.431443, 2001.

${ }^{23}$ van Albada, G.D., van Leer, B., and Roberts, W.W. A Comparative Study of Computational Methods in Cosmic Gas Dynamics, Astronomy and Astrophysics, 108, pp.76-84, 1982.

${ }^{24}$ van Leer, B., Towards the Ultimate Conservative Difference Scheme, A Second Order Sequel to Godunov's Method, Journal of Computational Physics, 32, pp.101-136, 1979. 